\title{
Explicit and Implicit Memory Loss in Aging
}

\author{
Richard E. Hicks ${ }^{1}$, Victoria E. Alexander ${ }^{1} \&$ Mark Bahr $^{1}$ \\ ${ }^{1}$ Bond University, Australia \\ Correspondence: Richard E. Hicks, Bond University, Australia. E-mail: rhicks@bond.edu.au
}

Received: May 5, 2018

Accepted: July 30, 2018

Online Published: August 16, 2018

doi:10.5539/ijps.v10n3p40

URL: https://doi.org/10.5539/ijps.v10n3p40

\begin{abstract}
How our memory is affected as we age has been given considerable attention over recent decades as we strive to understand the cognitive processes involved. Memory types have been identified as either explicit (declarative related to episodes or semantics) or implicit (non-declarative - related to procedures, habits, or earlier priming). Studies have identified likely age-related decline in explicit but not implicit memory though there are opposing results suggested from other studies. It is thought cognitive reserve capacities might explain any non-decline as aging individuals use alternative or additional pathways to 'remember'. This theory might be supported indirectly if older members remember material accurately but take longer to supply answers. In our current study we re-examined whether age-related differences in accuracy and speed of access in memory are present in both implicit and explicit memory processes and we increased the number of experimental age groups (from 2 to 3 ) most previous studies have compared just two groups (young, and old). With three groups (young, middle-old, and older aged groups) we can identify trends across the age range towards deterioration or preservation of memory. We examined sixty-six participants (49 females; 17 males) aged 18 to 86 years $(M=50.27, S D=21.06$ ) from South-Eastern Queensland and divided these into younger (18 to 46 years of age), middle old (50 to 64) and older aged $(65+)$ cohorts. Participants were administered tasks assessing implicit and explicit memory using computer presentations. Consistent with most prior research, no age differences were identified on accuracy in the implicit memory tasks (verbal and non-verbal, including priming), suggesting that memory for implicit material remains preserved. However, on the explicit memory tasks, older adults performed less accurately than the younger adults, indicative of decline in explicit memory as we age. The finding of a decline in explicit memory but no significant decline in implicit memory confirms most earlier research and is consistent with a view of modular decline rather than overall decline in memory with increasing age. In addition, differences found in speed of response in otherwise accurate implicit memory with older respondents significantly slower, suggests possible support for the cognitive reserve hypothesis.
\end{abstract}

Keywords: aging, cognitive decline, explicit memory, implicit memory, cognitive reserve

\section{Introduction}

How our memory is affected as we age has been given considerable attention over recent decades as we strive to understand the cognitive processes involved. Most developmental psychology text books today (e.g., Hoffnung et al., 2016; Sigelman \& Rider, 2018) discuss the background to memory and memory 'types' as they indicate how memory develops in the earliest ages after birth and deteriorates (e.g., forgetting, or not recognising previously known aspects or names) as we head towards the end of life. Memory types have been identified as either explicit memory ('declarative' - related to episodes or memory for events involving explicitly for example what we had for breakfast or items from lists of learned words, numbers or faces) or implicit memory ('non-declarative' - related to procedures, habits, or priming) (Sigelman \& Rider, 2018). Priming involves preparations for an event or task, such as preview of materials without intent to learn elements- learning occurring without effort to learn.

As part of the study of memory (and its decline with age) different cognitive and biological areas have been examined, not just the explicit and implicit memory processes which are the focus of this article. Some of these broader areas have included the numerous physiological and biological studies that include those addressing theories on memory loss (e.g., see Park \& Festini, 2016); the importance of nutrition in aging (Vauzour et al., 2017); neuronal effects as we age including effects in dementia (e.g., Kennedy et al., 2012; Nyberg et al., 1996; Wang et al., 2011); working memory loss (Wang et al., 2011); cognitive reserve in dealing with memory loss in ageing and dementia (e.g., Allen, Bruss, \& Damasio, 2005; Stern, 2009; Tucker \& Stern, 2011; 
Tucker-Drob, Johnson, \& Jones, 2009); and more. Some more specific studies have included memory decline for general vs specific details (e.g., Guerin, Robbins, Gilmore, \& Schachter, 2012; Alexander, Bahr, \& Hicks, 2015); and facial recognition and visual processing (e.g., Carbon \& Leder, 2005; Hicks, Alexander, \& Bahr, 2017). However, explicit vs implicit studies remain somewhat divided and we set about examining further what happens to these memory aspects as we age.

Most studies have identified likely age-related decline in explicit but not implicit memory suggesting that multiple senses are involved in memory and that memory is not part of a single system that impacts overall memory and general decline as we age. In addition to these studies there have been many studies that have examined age related differences in explicit and implicit memory and whether these processes are vulnerable to similar decline. For example, Graf (1990) identified what was then known about life-span changes in implicit and explicit memory, indicating the loss of explicit memory over time but the retention of implicit memories. There have been several studies both before and after the Graf (1990) paper and some of these are reviewed briefly in what follows, but it is also noted that not all current researchers believe the evidence is as strong as might appear to be the case for loss of explicit memory but not for implicit memory. For example, Ward and his colleagues have completed experimental studies supporting not modular decline but a single-system memory decline (an overall decline- e.g., Ward, Berry, \& Shanks, 2013). The current study examines this area further and reports a study we conducted on the relationship between explicit and implicit memory decline or otherwise, over three major age periods. We highlight some other earlier explicit-implicit studies first.

Studies that have identified evidence of age related cognitive decline in explicit memory include those of Eich, Murayama, and Knowlton (2014); Light, Singh, and Capps (1986); Schugens, Daum, Spindler, and Birbaumer (1997); and Spaan and Raaijmakers (2011); but there are a few (see next paragraph) that have suggested age-related implicit memory decline. Most emphasise no decline or limited decline. For example, Light et al. (1986) when investigating the effects of aging on implicit memory using a stem completion task, found that older adults were not significantly different from the younger aged cohort in remembering. This finding was supported by others including more recent studies- such as in Gopie, Craik, and Hasher's (2011) study that found older adults had better performance on the implicit word-fragment completion test than did younger adults and in Spaan and Raaijmakers'(2011) study using a priming and stem completion task (see a similar approach for the current study, outlined in Method, ahead). These found that priming was largely invariant with age (no differences over age ranges). These studies provide support for implicit memory remaining preserved.

However, Schugens et al. (1997) identified significant age-related decline on the stem completion task, testing implicit memory - the results arising, the authors suggested, by contamination from explicit memory. This conclusion was supported through a factor analysis which identified that priming (preparatory material that was not part of explicit learning requirements but involved in implicit memory studies) loaded onto a factor involving explicit memory. Schugens et al. (1997) suggested that visually presenting word stems might have acted as a retrieval cue and induced explicit memory processes. In addition, they suggested that the lack of time delay between tasks and use of instructions might also have induced the use of explicit strategies. Another, more recent, study by Dew and Giovanello (2010) also examined the effect of explicit memory processes as aiding implicit memory recall to explain the mixed findings over time on the explicit-implicit question. They found, however, in favour of differential (not general) age effects, with implicit memory being preserved from the age-decline process. That is, it seems the effects of any explicit information, on implicit learning, are limited.

There have been other attempts to tease out whether the research designs truly allow implicit memory strategies to be assessed independent of influences from explicit memory processes. Shum, O'Gorman, and Eadie (1999) assessed non-verbal implicit memory using the Shum Visual Learning Test (SVLT; Shum et al., 1999). On this task participants were provided a form of instruction that did not explicitly instruct them to learn the symbols, and used a different set of images and distractors - a process that was aimed at creating proactive interference and hence facilitating memory performance. (Proactive interference occurs when earlier learned materials distract individuals from learning new materials). Memory retention of the original material after the interference stage was then assessed. The authors (Shum et al., 1999) found that the individuals in the older age groups $(60-69 ; 70+)$ recognised significantly fewer characters than younger participants but also after the proactive interference stage. Decline in non-verbal implicit memory appeared to be occurring. So, Shum's study suggested implicit memory decline occurred. These findings were different from those of Light et al. (1986), and Schugens et al. (1997); and also of the recent findings by Spaan and Raaijmakers (2011). Because the possible decline was related to non-verbal material (in Shum's study) we set up our study to include both verbal and non-verbal implicit memory material with a view to clarifying whether the differences existed. 
Since much earlier research has not only identified poorer memory as we age but linked the loss of memory performance to slower speed in recall even when accurate, attention in research has also been given to this slowing effect in our current study. These effects have been noted in numerous studies (such as in Alexander, Bahr, \& Hicks, 2014; Birren, Woods, \& Williams, 1980; Eich et al., 2014; \& Shum et al., 1996). We included timed measures to examine the speed of recall in our further study to address the extent to which the slowing was evident across the age range. Links to the cognitive reserve hypothesis (cf., Tucker-Drob et al., 2009) were hoped to be made.

\subsection{Aims of the Current Study}

The current study investigated whether a decline occurs in accuracy and time taken (slowing) to process implicit memory materials (in the non-verbal domain and/or also in the verbal domain); and also examined similar decline in explicit memory (there was no debate about decline in explicit memory, so just the one experiment using non-verbal memory material was used- on memory for digits or numbers over given periods). A related aim in the current study was to assess whether age related cognitive decline is modular or generalised (specific, or overall- not limited to specific elements). If there is decline in explicit memory and implicit memory remains preserved, or if non-verbal but not verbal memory declines, either result provides evidence for modular decline (decline occurring non-uniformly, specifically, and not in an overall manner) rather than an overall decline in the aging brain. If decline is found to be modular and affects specific subsystems but not all systems, then interventions can be tailored to treat these specific subsystems. Therefore, in summary, our study aimed to assess whether there is dissociation between implicit and explicit memory processing; and also to assess whether there is dissociation between verbal and non-verbal implicit memory-which would also provide further evidence of modular decline. As a result evidence for attention to strengthening aspects that would benefit from training would be increased and hopefully strategies developed that would help those dealing with memory loss in normal aging and also help those dealing with non-normal aging as in dementia.

\section{Method}

\subsection{Participants}

Seventy participants from South-Eastern Queensland were recruited to participate in the study. A purposive sampling technique was used, following Babbie (2007) to provide balance across the three aged samples of interest in terms of the main characteristic desired- that is, of 'independent living healthy older adults'. Screening of the data lead to the final sample of 66 comprising of 49 females $(74.2 \%)$ and 17 males $(25.8 \%)$. The age of the sample ranged from 18 to 86 years $(M=50.27, S D=21.06)$. For highest education obtained, $40(60.6 \%)$ participants nominated high school, 17 (25.8\%) university, 5 (7.6\%) nominated T.A.F.E/college and 4 nominated primary school $(6.1 \%)$. From the participants, $36(30.45 \%)$ were currently taking medication for illnesses e.g., high blood pressure, high cholesterol, diabetes, vascular problems. The other 30 participants $(45.5 \%)$ were not currently taking medication. There were significantly more members taking medication in the older age groups. This is consistent with older age groups and as we screened for healthy independent living (that is, in the case of medication, the problems were under a good degree of control) we continued with the study. We also screened out any participants not meeting the criteria for independent living, for example, those with severe depression. Language background (in Chinese) may have been important in our study but there were no Chinese members in the groups. For more details on the make-up and balance of the sample see Alexander (2014).

\subsection{Instruments and Processes Used in Measurement}

There were three separate areas assessed as described in the subsequent paragraphs: non-verbal implicit memory, using Chinese characters (accuracy in recognition, and latency or time taken); verbal implicit memory, using common and rare words of 5 letters each (accuracy, and latency); and explicit memory, using digit span recall (accuracy and longest span recalled, and latency). A distractor stage was developed to assess the effects of proactive interference (that is the extent to which learned older materials distract the learning of new materials, in implicit memory). In the explicit learning/recall tasks there was not a distractor stage but clear explicit directions to learn the materials for recall. The distractor (proactive interference) stage was needed only in the implicit learning study as we needed the stage to be able to show whether learning (undirected) had occurred. We did not need a distractor stage in the explicit study. In the explicit experiment the evidence for whether learning had occurred was directly available (via the explicit directions regarding the learning of the material: this could not be the case in implicit learning, needing the distractor stage). We were interested in across-age differences if any, and the experimental design enabled us to answer questions on the aims of the study and to do so in detail (see Results including related tables). More detail follows on the three experiments. 


\subsection{Non-Verbal Implicit Memory}

\subsubsection{Materials/Instrument}

To assess non-verbal implicit memory, the Shum Visual Learning Test (SVLT; Shum et al., 1999) was used. The stimuli for each of the six learning phases consisted of 10 Chinese characters. The stimuli for the experimental component of the task were 20 Chinese characters. Ten of the characters presented were from the initial learning phase. The other 10 Chinese characters were also previously shown in the learning phase but with a stroke added or removed to act as distractors. Refer to Figure 1 below for examples of stimuli used in the task.
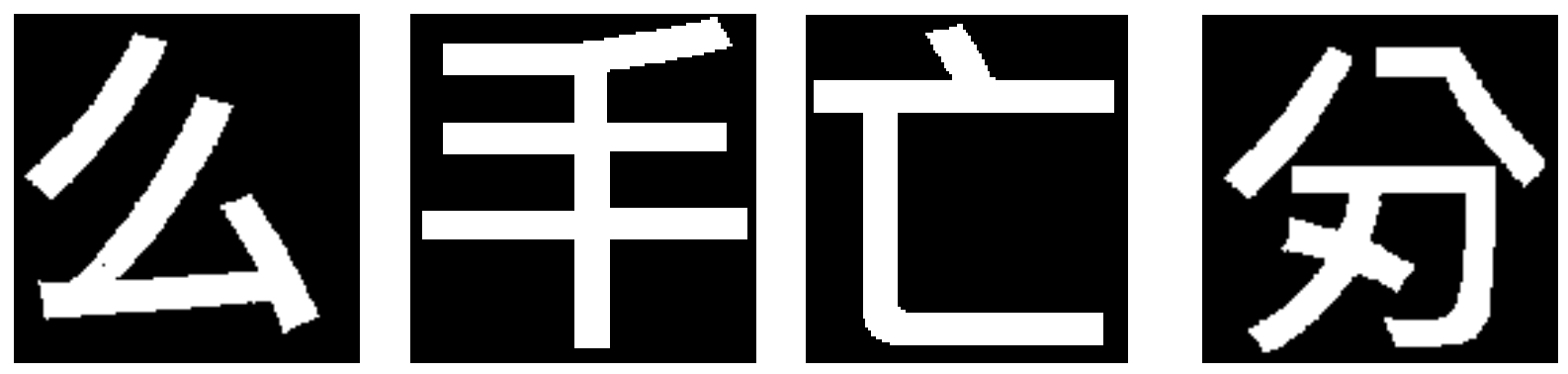

Figure 1. Selected Stimuli from the Non-Verbal Implicit Memory Task

\subsubsection{Process/ Procedures for the Non-Verbal Implicit Memory Task}

There was an initial learning phase- of the number of strokes in each symbol (5 trials involving exposure to the Chinese symbols), with a sixth trial with new symbols but the same instructions (to count the number of strokes in each symbol), and then two further trials involving whether learning of the original symbols themselves had occurred and had been retained or impeded following trial 6 (which acted to reduce proactive interference). More details follow.

For each of the first five trials, participants were instructed to count the strokes in each symbol (see Figure 1). The instructions were intended to distract participants from the intended nature of the task (remembering or learning to recognize the actual symbols: they were not informed of this aspect as this would have interfered with the dynamics of the study). On each of the trials, participants were presented the same Chinese characters in turn and required to count the strokes in the character. Each of the characters was presented for $3000 \mathrm{~ms}$ ( 3 secs) to ensure exposure and allow for implicit learning to occur. A blank time of $500 \mathrm{~ms}(1 / 2 \mathrm{sec})$ was presented between each Chinese character to allow the participant to reset prior to the presentation of the next word.

On Trial 6, participants were given the same instructions as the previous trials on the learning and test phase (that is, to count the number of strokes) but were presented a different set of characters. This manipulation was designed to release the individual from proactive interference and facilitate memory performance.

For the first five experimental phases (in Trials 7 and 8), participants were presented 20 Chinese symbols (characters) that were counterbalanced in order with ten of the characters presented being from the initial learning phase, and the remaining ten being from the learning phase but each having an extra stroke added or removed, designed to act as distractors. Participants were required to indicate whether they had seen or had not seen the symbol previously, by typing the appropriate key on the keyboard. Each of the characters was presented for $15,000 \mathrm{~ms}(15 \mathrm{sec})$ before the task timed out and the next character was presented. It is thought that presenting the same characters on each trial creates proactive interference, that is, interference with the learning of new materials. Participants were not provided the initial learning materials on the seventh and eighth trials, the experimental phase where memory was being assessed for the symbols (not the number of strokes). On both trials, participants were tested on the same set of characters as in the initial five trials. Trial 7 was designed to measure retention after interference. Following Trial 7, a 20-minute interval was provided, and Trial 8 followedTrial 8 was designed to assess retention after delay. For the $7^{\text {th }}$ and $8^{\text {th }}$ trials, participants were not provided a learning phase as that learning stage was designed within Trials 1-5.

\subsubsection{Comparing the Process for the Implicit and the Explicit Experiments}

The above section outlined the procedure regarding the implicit studies (described above in 2.3 and also in 2.4 below); the procedure regarding the explicit study ( 2.5 below) did not need the development of a separate set of memories that were clearly explicit in nature: instead the recall of material explicitly requested to be learned was needed. We chose the traditional measure of recall of sets of numbers ('digit span') for that experiment. We thus 
could assess, for both implicit and explicit learning circumstances, the degree of recognition or memory, that had occurred. The aim of the study, to assess accuracy and speed of the memory and whether declines occurred as we age, was accomplished by using the different age groups across the age span.

\subsection{Verbal Implicit Memory}

\subsubsection{Materials/Instrument}

To assess verbal implicit memory, a stem completion task was used. The stimuli for the vocabulary-learning phase consisted of 40 nouns each of 5 letters. Twenty of the words were high familiarity words and 20 were low familiarity words. The words for the stem completion task were taken from a subsample of 6000 words derived from a 100-thousand word-list based on the Corpus of Contemporary American English (e.g., Davies, 2011), the British Natural Corpus, Corpus of American Soap Operas and Corpus of Historical American English. The high frequency words (commonly used words) were selected with a dispersion of over 0.90 and total frequency of over 1050. The low frequency words (rare or uncommon use) were selected with a diversion of under 0.90 and total frequency under 250 (see Davies, 2011). Each of the words was matched for target word and syllable length.

\subsubsection{Process/ Procedures for the Verbal Implicit Memory Task}

In the initial learning phase participants were presented 40 words each consisting of 5 letters in length (e.g., 'braid') (example only, not one of the actual words). During the learning phase, participants could develop a general impression of the word- how familiar the word was to them. As this was an implicit task where learning is planned to be unintentional, participants were prescribed a specific task to distract them from the actual nature of the task. That is, they were instructed that words of varying levels of familiarity to most people would be shown to them briefly in turn and that they were to consider how familiar the words were to them. No other instructions were issued. Each of the words was presented for $2000 \mathrm{~ms}(2 \mathrm{sec})$ to allow time for implicit or incidental learning to occur. A $500 \mathrm{~ms}(1 / 2 \mathrm{sec})$ blank time was presented to allow the participant to reset prior to the presentation of the next word.

After the 40 words were presented, a new task was presented that involved a number search task and was presented prior to the experimental phase. In the number search task, participants were presented blocks of numbers and were required to search for the number 7. This was designed to provide a longer time period between the initial learning phase and the experimental phase and make the stem completion task more difficult.

In the experimental phase, participants were presented 50 trials. On each trial, a 3-letter stem (e.g., 'bra...') was presented being either the beginning of an "old word" presented from the learning phase, or of a "new word" that had not been seen previously. Each of the stems was presented with two question marks on the end (e.g., 'bra??') Participants were provided with $15,000 \mathrm{~ms}(15 \mathrm{sec})$ to respond by completing the five-letter word they considered they had seen previously (though not deliberately set out to learn), before the task would time out and the next item (stem) would be presented.

\subsection{Explicit Memory Task}

A similar process was followed in the explicit memory task, except that directions were clearly focused on learning the materials presented- in this case, a set of words, or of digits ('span') to be recalled. There was no need for an initial learning (exposure) phase or set of trials (as these were used to set up the implicit learning conditions); this was a straight, explicit, open learning experiment where digits recalled correctly (the span) and the latency (time taken) were assessed directly.

\subsubsection{Explicit Memory: Materials and Process}

To assess explicit memory, a computer administered digit span task was used. Participants were presented with random sequences of digits. Participants were administered a forward span task using a staircase method. Initial list length presented was three digits (repeating the digits 'forward', that is in order presented). Each successful reproduction of the stimulus string resulted in an increase in the stimulus list length by one digit. Each error resulted in a reduction of the list length by one digit. Participants were presented with 30 trials. The display time of the stimuli (that is, the series of digits to be recalled) was $3000 \mathrm{~ms}(3 \mathrm{sec})$, followed by a $2000 \mathrm{~ms}(2 \mathrm{sec})$ blank time. Participants had $10,000 \mathrm{~ms}(10 \mathrm{sec})$ to respond after which time the stimulus timed out, and the response was excluded from the analysis. There was a $1000 \mathrm{~ms}(1 \mathrm{sec})$ inter-stimulus interval. Span achieved was recorded as the average of the last 10 trials, that is trials or presentations $21-30$ of the 30 trials. 


\subsection{Statistical Design for each of the Three Memory Studies}

\subsubsection{Non-Verbal Implicit Memory}

A $3 \times 8 \times 2$ mixed factorial design was used. The independent and between subjects variable was Age (Young (18-49), Middle Old (50-64), and Older Adults (65 and above)). The independent variables Trial (Trials 1-8) and Target (Hit - Correct vs. Distractor - Miss) varied within subjects. The dependent variable was Recognition.

\subsubsection{Verbal Implicit Memory}

A 3 × 2 mixed factorial design was used. The independent and between subjects variable was Age [Young (18-49), Middle Old (50-64), and Older Adults (65 and above)]. The independent variable Frequency (High vs. Low Frequency Words) varied within subjects. The dependent variables were Accuracy and Latency.

\subsubsection{Explicit Memory}

A between subjects design was used. The independent variable was Age (Young (18-49), Middle Old (50-64), and Older Adults (65 and above). The dependent variables were Mean Span (length recalled) and Mean Latency (time taken).

\subsection{Our Research Questions and Hypotheses}

Based on our review of the literature we hypothesized that memory for implicit material (both verbal, and non-verbal) would remain preserved over the three lifespan ages we studied but explicit memory would decline. We also anticipated that the older age groups would take longer (latency) to achieve recall: that is there would be an effect of age on latency. We also hypothesized that further support for modular rather than general cognitive decline occurring as we age would result, thus confirming earlier findings on explicit and implicit memory.

\section{Results}

\subsection{Preliminary Analyses and Data Cleaning}

Initial data checking using IBM SPSS Statistics 21 confirmed that the data met the assumptions for Multivariate analysis of variance (MANOVA) and Analysis of variance (ANOVA).

\subsubsection{Non-Verbal Implicit Memory}

Prior to analysis, the assumptions of MANOVA were tested. The assumption of sample size was met. Violations of normality for all levels of the DV were identified using Kolmogorov-Smirnov $(p<.05)$. However, MANOVA is robust to moderate violations of normality with larger sample sizes. One participant was removed (a Chinese speaker whose language knowledge may have affected the results). An additional participant was removed as the task was not completed (no response on Trial 7). Two additional multivariate outliers were removed. The data were not multi-collinear. The assumption of homogeneity of variance-covariance matrices was met. No missing values were identified.

\subsubsection{Verbal Implicit Memory}

Prior to analysis, the assumptions of MANOVA were tested. The assumption of sample size was met. Violations of normality for all levels of both DVs were identified except for Accuracy of High Frequency Words. However, MANOVA is able to compensate for moderate violations in normality with larger sample sizes. It was identified that one younger adult, two middle old, and four older adults timed out on the task for low frequency words. Therefore, a mean imputation method was used and means of the young and middle old sample were used for the participants respectively. The four individuals in the older adult sample were imputed using 3 standard deviations above their mean as it was assumed they had significant decrement on the task. An alternative method would be to exclude the older adults with missing data. However, as they were unable to complete the low frequency words at all, this might indicate that that they are showing more signs of cognitive decline than other older adults and therefore are a cohort of interest. The data were linear. As MANOVA is able to compensate for a few outliers in larger sample sizes, no outliers were removed from the analysis. Sphericity was assumed. The data were not multi-collinear. Box's $M$ was violated for Latency $[F(6,89420)=12.24, M=76.99, p<.001]$. As Box's $M$ was violated, a more conservative Pillai's criterion was used to interpret multivariate significance.

\subsubsection{Explicit Memory: Digit Span}

Prior to running analyses, the assumptions of ANOVA were tested. The data was normal. Levene's Test indicated equal variances. No missing data was identified. 


\subsection{Main Analyses}

\subsubsection{Non-Verbal Implicit Memory}

A mixed factorial MANOVA was conducted to assess the effect of Age, Trial and Target on Recognition. $\alpha$ was set at .05 apriori. There was a significant Age x Trial x Target interaction $\left(F(14,116)=1.84, p=.040\right.$, partial $\eta^{2}$ $=.18$, power $=.91$ ). To assess the 3-way interaction, the data was split by Age and Target. It was identified that there was a significant effect of Hits over the eight trials for the Younger Adults $(F(7,161)=5.65, p=.002$, partial $\eta^{2}=.70$, power $=.98$ ). There was a significant effect of Hits over the eight trials for Middle Old Adults $\left(F(7,140)=5.78, p=.003\right.$, partial $\eta^{2}=.74$, power $\left.=.97\right)$. In addition, there was a significant effect of Hits over the eight trials for Older Adults $\left(F(7,140)=4.91, p=.006\right.$, partial $\eta^{2}=.71$, power $\left.=.95\right)$. As can be seen in Table 1 below, there is some evidence of an increase in hit recognition for all cohorts over the eight trials.

Table 1. Average Number of Hits Recognised over the Eight Trials for Young Old, Middle Old, and Older Adults

\begin{tabular}{llll}
\hline \multirow{2}{*}{ Age Group } & Young Old & Middle Old & Older Adults \\
& $M(s d)$ & $M(s d)$ & $M(s d)$ \\
\cline { 2 - 3 } Trial 1 & 6.54 & 6.52 & 6.29 \\
& $(1.93)$ & $(1.94)$ & $(1.88)$ \\
Trial 2 & 7.00 & 8.05 & 7.71 \\
& $(2.02)$ & $(1.72)$ & $(1.23)$ \\
Trial 3 & 6.71 & 7.71 & 7.38 \\
& $(2.53)$ & $(1.55)$ & $(1.88)$ \\
Trial 4 & 8.17 & 7.76 & 7.90 \\
& $(1.79)$ & $(1.92)$ & $(1.34)$ \\
Trial 5 & 8.08 & 8.19 & 7.81 \\
& $(2.00)$ & $(1.50)$ & $(1.83)$ \\
Trial 6 & 6.58 & 5.90 & 6.43 \\
& $(1.77)$ & $(2.51)$ & $(2.52)$ \\
Trial 7 & 8.88 & 8.81 & 8.52 \\
& $(1.54)$ & $(1.47)$ & $(1.78)$ \\
Trial 8 & 9.08 & 9.38 & 8.95 \\
& $(1.35)$ & $(.87)$ & $(1.53)$ \\
\hline
\end{tabular}

There was a significant effect of Distractors over the eight trials for the Younger Adults $(F(7,161)=10.60, p$ $<.001$, partial $\eta^{2}=.82$, power approaching 1). There was also a significant effect of Distractors over the eight trials for Middle Old Adults $F(7,140)=6.95, p=.001$, partial $\eta^{2}=.78$, power $\left.=.99\right)$. In addition, there was a significant effect of Distractors over the eight trials for Older Adults $\left(F(7,140)=6.51, p=.002\right.$, partial $\eta^{2}=.77$, power $=.98$ ). As can be seen in Table 2 below, the three cohorts also each recognised (had learned) significantly more distractors across the eight trials. 
Table 2. Average Number of Distractors Recognised over the Eight Trials for Young Old, Middle Old, and Older Adults

\begin{tabular}{llll}
\hline \multirow{2}{*}{ Age Group } & Young Old & Middle Old & Older Adults \\
& $M(s d)$ & $M(s d)$ & $M(s d)$ \\
\cline { 2 - 3 } Trial 1 & 3.29 & 4.38 & 4.57 \\
& $(1.65)$ & $(2.04)$ & $(1.94)$ \\
Trial 2 & 4.75 & 5.76 & 5.43 \\
& $(1.73)$ & $(1.81)$ & $(1.91)$ \\
Trial 3 & 5.08 & 5.29 & 6.62 \\
& $(2.22)$ & $(2.31)$ & $(1.94)$ \\
Trial 4 & 5.13 & 5.52 & 6.71 \\
& $(1.66)$ & $(1.81)$ & $(1.90)$ \\
Trial 5 & 6.17 & 5.81 & 6.95 \\
& $(2.60)$ & $(2.11)$ & $(1.56)$ \\
Trial 6 & 4.54 & 4.52 & 4.95 \\
& $(2.02)$ & $(2.56)$ & $(2.33)$ \\
Trial 7 & 7.25 & 7.57 & 7.95 \\
Trial 8 & $(2.23)$ & $(1.89)$ & $(1.62)$ \\
& 7.46 & 7.98 & 8.00 \\
\hline
\end{tabular}

3.2.1.1 Proactive Interference

As may be seen in Table 1 above, the three cohorts show an increase in learning demonstrated by increased frequencies of hits across the five learning trials. However, they also show an increase in the learning of distractors suggesting some evidence of proactive interference across the first five trials (Refer to Table 2 above). As can be seen in Table 2 and 3, there is a drop in hit and distractor recognition on the release from proactive interference trial (Trial 6) with scoring returning almost to baseline responding. Following the release from proactive interference trial, there is an increase in hit and distractor recognition on Trials 7 and 8 for all cohorts.

There was also a significant Age x Target interaction $\left(F(2,63)=3.87, p=.026\right.$, partial $\eta^{2}=.11$, power $\left.=.68\right)$. To assess the influence of Age within levels of Target, Trial was collapsed and a series of ONEWAY between groups univariate ANOVAs was conducted. $\alpha$ was set at .05 apriori. Rather than taking the average of the eight trials, which include the learning and release from interference phases, only Trials 7 and 8 were analysed. On Trial 7 there was no significant effect of Age on Hits $F(2,63)=.30, p=.744$, partial $\eta^{2}=.01$, power $=.10$ ) or Distractors $F(2,63)=.72, p=.495$, partial $\eta^{2}=.02$, power $\left.=.17\right)$. On Trial 8 , there was again no significant effect of Age on Hits $F(2,63)=.62, p=.542$, partial $\eta^{2}=.02$, power $\left.=.05\right)$ or Distractors $(F(2,63)=.55, p$ $=.649$, partial $\eta^{2}=.01$, power $=.12$ ).

\subsubsection{Verbal Implicit Memory}

A mixed factorial MANOVA was conducted to assess the effect of Age (Young Old (18-49), Middle Old (50-64), and Older Adults (65+)), and Frequency (High vs. Low) on Accuracy. $\alpha$ was set at .05 apriori. There was no significant Age x Frequency interaction $\left(F(2,63)=1.46, p=.240\right.$, partial $\eta^{2}=.04$, power $\left.=.31\right)$. As the interaction was not significant, each of the main effects was considered. There was a significant main effect of Frequency, with high frequency words completed more accurately than low frequency words $(F(1,63)=286.31$, $p<.001$, partial $\eta^{2}=.82$, power approaching 1). It was identified that high frequency words were completed more accurately than low frequency words. However, there was no significant main effect of $\operatorname{Age}(F(2,63)=$ $2.27, p=.111$, partial $\eta^{2}=.07$, power $=.45$ ). Although there was no significant effect of Age, there is evidence of a downward trend from young to older adults (Refer to Table 3 below). A polynomial contrast was also conducted on Age. Although there was no significant quadratic decline $(p=.859)$, the linear contrast was significant $(p=.034)$. This suggests there is some evidence of a monotonic decline. 
Table 3. Average Number of High and Frequency Words Correct for Young Old, Middle Old, and Older Adults

\begin{tabular}{llll}
\hline \multirow{2}{*}{ Age Group } & Young Old & Middle Old & Older Adults \\
& $M(s d)$ & $M(s d)$ & $M(s d)$ \\
\cline { 2 - 4 } High Frequency Words & 10.46 & 9.10 & 8.52 \\
& $(3.02)$ & $(2.64)$ & $(2.32)$ \\
Low Frequency Words & 3.71 & 3.59 & 3.05 \\
& $(2.12)$ & $(1.83)$ & $(2.62)$ \\
\hline
\end{tabular}

3.3.2 Latency (Time Taken to Results)

A mixed factorial MANOVA was conducted to assess the effect of Age (Young Old (18-49), Middle Old (50-64), Older Adults (65+) and Frequency (High vs. Low) on Latency. $\alpha$ was set at .05 apriori. As Box's $M$ had been violated, a more conservative Pillai's criterion was used to interpret multivariate significance, unless otherwise indicated

There was a significant Age x Frequency interaction $\left(F_{\text {pillai's }}(2,63)=7.57, p=.001\right.$, partial $\eta^{2}=.19$, power $=.94$ ). To assess the effect of Age on levels of Frequency, a series of univariate ANOVAs was conducted. $\alpha$ was set at .05 apriori. There was a significant effect of Age on Latency to Complete High Frequency Words, with older adults slower to complete high frequency words than young and middle old adults $(F(2,63)=14.87, p$ $<.001$, partial $\eta^{2}=.32$, power approaching 1). To investigate group differences between Age, a series of post hoc analyses was conducted using Tukey's HSD with $\alpha=.05$. There was a significant difference between the young and older adults, with older adults slower to complete high frequency words than young adults. In addition, there was a significant difference between the young and older middle old adults, with older adults slower to complete high frequency words than middle old adults. However, there was no significant difference between young and middle old adults. Refer to Table 4 below.

Table 4. Average Latency (ms) for High and Low Frequency Words for Young Old, Middle Old, and Older Adults

\begin{tabular}{llll}
\hline \multirow{2}{*}{ Age Group } & Young Old & Middle Old & Older Adults \\
& $M(s d)$ & $M(s d)$ & $M(s d)$ \\
\cline { 2 - 4 } High Frequency Words & $2228^{\mathrm{a}}$ & $3048^{\mathrm{b}}$ & 4318 \\
& $(867)$ & $(902)$ & $(1867)$ \\
Low Frequency Words & $2767^{\mathrm{a}}$ & $3487^{\mathrm{b}}$ & 7441 \\
& $(1219)$ & $(1278)$ & $(4713)$ \\
\hline
\end{tabular}

NB: a - Difference between young and older adults, $b$ - Difference between middle and older adults

In addition, there was a significant effect of Age on Latency to Complete Low Frequency Words, with older adults slower to complete low frequency words than young and middle old adults $(F(2,63)=16.96, p<.001$, partial $\eta^{2}=.35$, power approaching 1). To investigate the differences between Age, a series of post hoc analyses were conducted using Tukey's HSD with $\alpha=.05$. There was a significant difference between the young and older adults, with older adults slower to complete low frequency words than young adults. In addition, there was a significant difference between middle and older adults, with older adults slower to complete low frequency words than middle old adults. However, there was no significant difference between young and middle adults. Refer to Table 4 above.

\subsubsection{Explicit Memory}

A ONEWAY between groups ANOVA was run to assess the effect of Age (Young Old (18-49), Middle Old (50-64), and Older Adults (65+)) on Mean Span. $\alpha$ was set at .05 apriori. There was an effect of Age on Mean Span, with older adults recalling fewer digits than younger adults $\left(F(2,63)=6.82, p<.001\right.$, partial $\eta^{2}=.18$, power $=.91)$. Post hoc analysis was conducted using Tukey's HSD $(\alpha=.05)$. There was a significant difference between young and older adults with older adults recalling fewer digits than young adults. However, there were no significant differences between middle old and older adults or young and middle old adults Refer to Table 5 below. 


\subsubsection{Latency (Time Taken)}

A ONEWAY between groups ANOVA was also run to assess the effect of Age (Young Old (18-49), Middle Old (50-64), and Older Adults (65+)) on Mean Latency. $\alpha$ was set at .05 apriori. There was no significant difference effect of Age on Mean Latency $\left(F(2,63)=1.25, p=.294\right.$, partial $\eta^{2}=.04$, power $\left.=.26\right)$. Refer to Table 5 .

Table 5. Mean Span and Latency (ms) by Age Group: Young Old, Middle Old, and Older Adults

\begin{tabular}{llll}
\hline \multirow{2}{*}{ Span and Latency Variables } & $\begin{array}{l}\text { Young Old } \\
M(s d)\end{array}$ & $\begin{array}{l}\text { Middle Old } \\
M(s d)\end{array}$ & $\begin{array}{l}\text { Older Adults } \\
M(s d)\end{array}$ \\
\cline { 2 - 4 } Mean Span & $7.25^{\mathrm{a}}$ & 6.98 & 6.01 \\
& $(1.08)$ & $(1.24)$ & $(1.19)$ \\
Mean Latency & 6982 & 7401 & 7367 \\
& $(1026)$ & $(716)$ & $(1201)$ \\
\hline
\end{tabular}

NB: a - Young different from older adults

\section{Discussion}

\subsection{Overview}

Do we continue to learn as we age? Our study (or series of studies) re-assessed whether accuracy in memory (both implicit and explicit memory) deteriorates markedly with age and whether we continue to learn and remember new material; it aimed to clarify, as most studies have indicated, whether explicit memory deteriorates while implicit memory does not; it also sought to determine whether accuracy in non-verbal and verbal implicit memory processes declined at different rates (such differences supporting 'modular decline': the decline in modes or processes in recall); and we also aimed in the study to determine whether we were slower in processing as we aged ('latency' in memory recall) and to compare such latency with accuracy in recall.

We thus divided the studies into two major segments: accuracy in memory across age groups, and latency (time taken) in recall of the target material across the age groups. We developed three different studies emphasizing accuracy and latency in dealing with materials requiring (separately) verbal memory, non-verbal memory, and explicit (digit span) memory. The design thus enabled us to address the issues we were interested in: in regards to decline or otherwise in memory accuracy, and also decline or otherwise in time taken in processing information. The results are placed in context and discussed in the following sections, first in regard to accuracy in memory retention across the three separate studies (verbal memory; non-verbal memory; explicit -'digit span'- memory) and then in regard to latency.

\subsection{Accuracy in Memory (Non-Verbal Implicit; Verbal Implicit; and Explicit Studies)}

From the research (e.g., Light et al., 1986; Spaan \& Raaijmakers, 2011), there was evidence to suggest that memory for implicit material would remain preserved. Therefore, it was predicted that no age differences would be identified on the implicit memory measures. We examined both non-verbal and verbal memory aspects.

From the measure of non-verbal implicit memory, there was a significant age by target interaction. However, when the interaction was split by target, it was found that older adults had similar performances to young and middle old adults on the recognition of hits and distractors (in accuracy in memory). As no decline was observed in hit recognition, this provides evidence that non-verbal implicit memory remains preserved from aging, consistent with the majority of earlier studies (such as Light et al., 1986, and Spaan \& Raaijmakers, 2011) but not consistent with the research of Shum et al. (1999) (who found older adults had significantly less target recognition than younger adults). A significant three way-interaction between age, trial and target was also observed in our results. So older members did less well in learning and retention in memory- but see next paragraph for a significant observation.

When the data was split by age and target, it was found that all groups increased in hit recognition (accuracy) across the eight trials. This suggests that all cohorts, including the older adults, showed learning over the trials. In addition, all groups increased in distractor recognition over trials. This suggests all cohorts were also more likely to learn distractors as well as hits. In terms of the non-verbal implicit memory study therefore, we concluded that implicit memory recall remains relatively unaffected by age. Would verbal implicit memory and explicit memory aspects yield similar results? Next.

Regarding verbal implicit memory, older adults had similar accuracy to the younger cohorts on the stem completion task, the measure of verbal implicit memory involving recall of a previously seen but not purposely 
learned word or element. This suggests that both younger and older adults had activation of relevant networks and were able to complete the word stems successfully. Moreover, it also suggests that older adults had sufficient encoding of the material despite not being instructed explicitly to learn the material. This provides indication that implicit memory of a verbal nature also remains preserved from aging. Moreover, there was an effect of frequency and that high frequency words (words commonly used by many people) were identified more accurately than low frequency words (words less well used or familiar). As an aside, this might suggest that low frequency words would be more effective in the detection of age related cognitive decline. Again, overall, we concluded that there was support for verbal implicit memory being maintained across the age span, though some attention to strategies we can use to aid recognition and recall of low frequency words may be needed. Some aspects hold up in verbal memory, others may not.

Regarding explicit memory (that is, involving specific directions to learn and recall material) we found that, consistent with earlier studies older adults had significantly lower span recall (were less accurate) than younger adults. This provides evidence of explicit memory decline.

However, as the decrease in span was small (average of 1 digit), this further suggests that explicit memory in the form of span recall is not as severe as has been suggested compared to other domains of cognition and that further studies are needed to confirm our findings (though significant). This is because span recall studies are usual in this area but given the small difference and therefore the decline being of less impact, further research is needed to sort out the actual elements associated with explicit decline. In addition, as discussed next, there was no significant effect of age on latency or time taken to results - different from what we have seen in earlier studies. It is possible that the older adults are making associations from numbers they have previously stored in memory, and therefore are not as affected in this area as may be thought. Implications for reducing decline and/or for rehabilitative studies are that accessing or using materials that are familiar at least to some extent will improve recall. Nevertheless, we did find significant decline in the explicit memory of those in the older age group.

\subsection{Latency/ Time-Taken}

Although no significant differences between cohorts on the effect of age on accuracy were identified, there was an effect of age on latency. Of interest, there was an age by frequency interaction (frequently used or familiar words were recalled more readily than less frequently used words). When the interaction was split by frequency, older adults were significantly slower on high and low frequency word recognitions than young and middle old adults. On the high frequency word latency data there was evidence of a linear decline. However, on the low frequency (rare) words there was evidence of an accelerated decline in the older adults. Again, this provides indication that low frequency words are more effective in the detection of age related cognitive decline.

As older adults did not decline on accuracy but had an increase in latency (time taken), this also provides some evidence for the cognitive reserve hypothesis. The cognitive reserve hypothesis claims that as direct pathways to knowledge become impaired, older adults may compensate by using redundant pathways (Stern, 2009). Subsequently, the use of alternative pathways results in higher latencies (longer time taken) in the retrieval of information. As there is an increase in latency but no apparent accompanying decrease in accuracy, this also provides some evidence of dissociation between accuracy and latency.

\subsection{The Modular Decline Model}

A further aim was to assess whether age related cognitive decline is modular or generalised. As decline was found in explicit memory but implicit memory remained preserved, this provides further evidence for dissociation of memory processing and for modular decline with increasing age. However, no dissociation between verbal implicit memory and non-verbal implicit memory processes was observed. Further, support of modular rather than overall general decline is suggested by the dissociations observed between recognition (accuracy) and latencies in these memory studies.

\subsection{Limitations}

There are always limitations in studies despite the care in design that may be taken. First, the sample may be biased in some way. Even though the major emphases in our three studies were on assuring reasonable comparability of the groups on the ability to lead independent healthy lives in the community, other elements may have influenced the results in unknown ways. For example, we requested medication information and as anticipated the older age group members consistently used more medication to aid their healthy living. Though we screened out those involved in severe mental health issues and related medications (therefore not able to live successful independent lives) there still may have been effects. We did not have sufficient numbers in our three 
samples to test against any effects on memory of the various mainly physical medications referred to. Further specialized medical studies would be needed for clarification of likely effects. However, given the screening processes used (and the consistency of most of our results with those of previous studies) we hazard to consider our results are robust across the populations studied. Again, further studies would help clarify these aspects.

\subsection{Implications and Conclusion}

Implications for treatment include targeting strategies that help individuals process materials more easily. As we age implicit memory remains largely unaffected. Therefore, we can have more confidence in older individuals being able to learn implicitly (naturally, without trying) though they may be slower than younger individuals. What might help training of memory and its rehabilitation is attention to the familiar: as already seen in some studies in Alzheimer's where return to the familiar helps in strong recuperative effects (e.g., Son, Therrien, \& Whall, 2002). For example, one implication from the lessened impact on the ability to recall digit span numbers (as representative of explicit memory processing), is that it is the familiar that may be assisting cognitive reserve development and new learning. Let us see what we can develop in further studies that help us to examine this possibility. In the mean-time our results confirm that there are differences in explicit and implicit memory loss as we age, consistent with the emphasis in most earlier studies on implicit and explicit memory, and supportive of the modular decline theory rather than overall general decline.

\section{References}

Alexander, V.E. (2014). Age-related decline: Detecting mild cognitive impairment. Unpublished Doctoral thesis, Bond University, Gold Coast, Australia

Alexander, V., Bahr, M., \& Hicks, R. (2015). Ability to recall specific detail and general detail (gist) in young old, middle old, and older adults. Psychology, 6(16), 2071-2080. https://doi.org/10.4236/psych.2015.616202

Alexander, V., Bahr, M., \& Hicks, R. (2014). Emotion recognition and verbal and non-verbal memory changes among older adults: Is decline generalised or modular? GSTF Journal of Psychology, 1(2), 14-21. https://doi.org/10.5176/0000-0002_1.2.14

Allen, J.S., Bruss, J., \& Damasio, H. (2005). The aging brain: the cognitive reserve hypothesis and hominid evolution. American Journal of Human Biology, 17(6), 673-89. https://doi.org/10.1002/ajhb.20439

Babbie, E. (2007). The Practice of Social Research. (1 $1^{\text {th }}$ Ed.). Belmont, CA: Wadsworth Cengage Learning.

Birren, J.E., Woods, A.M., \& Williams, M.V. (1980). Behavioural slowing with age: Causes, organization, and consequences. In Poon, L.W. (Ed.) Aging in the 1980s: Psychological Issues (pp. 293-308). Washington, DC. American Psychological Association. https://doi.org/10.1037/10050-021

Carbon, C.C., \& Leder, H. (2005). When feature information comes first! Early processing of inverted faces. Perception, 34, 1117-1134. https://doi.org/10.1068/p5192

Davies, M. (2011). Word frequency data from the Corpus of Contemporary American English (COCA). Retrieved from http://www.wordfrequency.info

Dew, I.T.L., \& Giovanello, K.S. (2010). Differential age effects for implicit and explicit conceptual associative memory. Psychology of Aging, 25(4), 911-921. https://doi.org/10.1037/a0019940

Eich, T.S., Murayama, K., Castel, A.D., \& Knowlton, B.J. (2014). The dynamic effects of age-related stereotype threat on explicit and implicit memory performance in older adults. Social Cognition, 32(6), 559-570. https://doi.org/10.1521/soco.2014.32.6.559

Gopie, N., Craik, F.I.M., \& Hasher, L. (2011). A double dissociation of implicit and explicit memory in younger and older adults. Psychological Science, 22(5), 634-640. https://doi.org/10.1177/0956797611403321

Graf, P. (1990). Lifespan changes in implicit and explicit memory. Bulletin of the Psychonomic Society, 28(4), 353-358. https://doi.org/10.3758/BF03334041

Guerin, S.A., Robbins, C.A., Gilmore, A.W., \& Schacter, D.L. (2012). Retrieval failure contributes to gist-based false recognition. Journal of Memory and Language, 66, 68-78. https://doi.org/10.1016/j.jml.2011.07.002

Hicks, R.E., Alexander V., \& Bahr, M. (2017). Facial recognition and visual processing as we age: Using the Thatcher Illusion with famous and non-famous faces. International Journal of Psychological Studies, 9(2), 26-36. https://doi.org/10.5539/ijps.v9n2p26 
Hoffnung,M., Hoffnung, R., Seifert, L., Hine, A., Ward, L., Pause, C., Swabey, K., Yates, K., \& Burton Smith, R. (2016). Lifespan Development $\left(3^{\text {rd }}\right.$ Australasian edition). Stafford, Queensland: Wiley

Kennedy, K., Rodrigue, K., Devous, M., Sr Hebrank, A., Bischof, G., \& Park, D. (2012). Effects of beta-amyloid accumulation on neural function during encoding across the adult lifespan. Neuroimage, 62, 1-8. https://doi.org/10.1016/j.neuroimage.2012.03.077

Light, L.L., Singh, A., \& Capps, J.L. (1986). Dissociation of memory and awareness in young and older adults. Journal of Clinical and Experimental Neuropsychology, 8(1), 62-74. https://doi.org/10.1080/01688638608401297

Nyberg, L., Blackman, L., Erngrund, K., Olofsson, U., \& Nilsson, L-G. (1996). Age differences in episodic memory, semantic memory, and priming: relationships to demographic, intellectual, and biological factors.

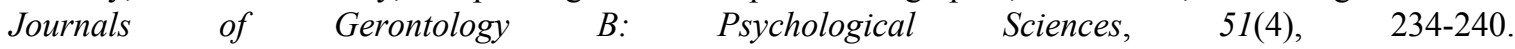
https://doi.org/10.1093/geronb/51B.4.P234

Park, D.C., \& Festini, S.B. (2017). Theories of memory and ageing: A look at the past and a glimpse of the future. Journals of Gerontology B: Psychological Sciences, 72(1), 82-90. https://doi.org/10.1093/geronb/gbw066

Schugens, M.M., Daum, I., Spindler, M., \& Birbaumer, N. (1997). Differential effects of ageing on explicit and implicit memory. Aging, Neuropsychology, and Cognition, 4(1), 33-44. https://doi.org/10.1080/13825589708256634

Shum, D.H.K., O'Gormon, J.G., \& Eadie, K. (1999). Normative data for a new memory test: The Shum Visual Learning Test. The Clinical Neuropsychologist, 13(2), 121-135. https://doi.org/10.1076/clin.13.2.121.1967

Sigelman, C.K., \& Rider, E.A. (2018). Life-Span Human Development (Ed. 9). Cengage Learning US. ISBN: 9781337100731.

Son, G-R., Therrien, B., \& Whall, A. (2002). Implicit memory and familiarity among elders with dementia. Journal of Nursing Scholarship, 34(3), 263-267. https://doi.org/10.1111/j.1547-5069.2002.00263.x

Spaan, E.J.P., \& Raaijmakers, J.G.W. (2010). Priming effects from young-old to very old age on a word-stem completion task: Minimizing explicit contamination. Aging, Neuropsychology, and Cognition, 18, 86-107. https://doi.org/10.1080/13825585.2010.511146

Stern, Y. (2009). Cognitive reserve. Neuropsychologia, 47, 2015-2028. https://doi.org/10.1016/j.neuropsychologia.2009.03.004

Tucker, A.M., \& Stern, Y. (2011). Cognitive reserve in ageing. Current Alzheimer Research, 8, 354-360. https://doi.org/10.2174/156720511795745320

Tucker-Drob, E.M., Johnson, K.E., \& Jones, R.N. (2009). The Cognitive Reserve Hypothesis: A Longitudinal Examination of Age-Associated Declines in Reasoning and Processing Speed. Developmental Psychology, 45(2), 431-446. https://doi.org/10.1037/a0014102

Vauzour, D., Cambrubi-Robles, M., Miquel-Kergoat, S., ... \& Ramirez, M. (2017). Nutrition for the ageing brain: Towards evidence for an optimal diet. Ageing Research Reviews, 35, 222-240. https://doi.org/10.1016/j.arr.2016.09.010

Wang, M., Gamo, N., Yang, Y., Jin, L. Wang, X-J.... \& Arnsten, A. (2011). Neuronal basis of age-related working memory decline. Nature, 476(7359), 210-213. https://doi.org/10.1038/nature10243

Ward, E.V., Berry, C.J., \& Shanks, D.R. (2013). Age effects on explicit and implicit memory. Frontiers in Psychology, 4, 639-655. https://doi.org/10.3389/fpsyg.2013.00639

\section{Copyrights}

Copyright for this article is retained by the author(s), with first publication rights granted to the journal.

This is an open-access article distributed under the terms and conditions of the Creative Commons Attribution license (http://creativecommons.org/licenses/by/4.0/). 